\title{
THE IMPACT OF SOFT-HANDOFF ON THE DOWNLINK - CAPACITY OF MOBILE CDMA SYSTEMS
}

\author{
André Noll Barreto and João Célio Brandão \\ Rua Marquês de S. Vicente, 225 \\ 22453-900, Rio de Janeiro - RJ \\ E-mail : noll@ifn.et.tu-dresden.de \\ jcelio@cetuc.puc-rio.br
}

\begin{abstract}
Resumo - O uso de soft-handoff pode ter um impacto substancial no enlace de descida de sistemas CDMA, reduzindo sua capacidade. Isto depende fortemente dos parâmetros utilizados no procedimento de handoff. Neste artigo avaliamos este impacto para diversos conjuntos de valores de parâmetros. Inicialmente estimamos a capacidade do sistema e a possível influência do soft-handoff em termos do número médio de estaçōes bases simultaneamente em contato com uma estação móvel. Em seguida determinamos mais precisamente o efeito de algoritmos de handoff e de parâmetros específicos. É obtida uma solução analítica para um sistema CDMA genérico e é simulado o procedimento de handoff da norma IS-95 com o objetivo de de estimar o tráfego efetivo suportado pelo sistema
\end{abstract}

\begin{abstract}
The use of soft-handoff may have a substantial impact on the downlink of cellular CDMA systems, by reducing its capacity. This depends heavily on the parameters used in the handoff procedure. In this paper we estimate this impact with several sets of parameter values. First we estimate the system capacity and the possible influence of softhandoff in terms of the average number of base stations simultaneously in contact to a mobile station. We then proceed to determine more precisely the effect of particular handoff algorithms and parameters. An analytical solution for a general CDMA system is derived and the IS-95 handoff procedure is simulated in order to estimate the effective traffic carried by the system
\end{abstract}

Keywords: CDMA, handoff, cellular communications.

\section{INTRODUCTION}

Soft-handoff is a well known technique used in mobile CDMA systems[1,2], which allows a mobile station to communicate with more than one base station simultaneously during handoff. This makes a seamless handoff possible, besides providing a better signal quality due to macro-diversity [1].

The employment of this technique has very little impact on the capacity of the uplink, since the same signal is transmitted by the mobile station, whether it is in soft-handoff or not. However, the same will not be true for the downlink. In this case, the same signal will be transmitted by two or more base stations, thus increasing the overall interference and consequently decreasing the capacity.
It is generally considered that the uplink capacity is much smaller than the downlink capacity, and that the former is thus the limiting resource in CDMA systems. The use of softhandoff however, can reduce the capacity of the downlink to such an extent that it becomes the limiting factor, depending on the handoff procedure and parameters. This situation is highly undesirable, and it is therefore interesting to determine the real effect of the soft-handoff in the capacity, and its dependence on the handoff parameters.

The effect of soft-handoff in CDMA systems has been studied recently in several papers $[3,4,5]$. In $[3,4]$ the margin required to meet a constant call reliability is determined, using both soft and hard handoff. In [3] an analycical solution is derived for the uplink, while in [4] results are obtained through simulation considering the handoff procedures from GSM and IS-95. In [5] an analysis of the effect of soft handoff in the downlink capacity was made considering only the overlapping regions between different cells. In this paper we present an analysis of the downlink capacity with soft handoff, considering a propagation model that includes path loss and shadowing, as well as the influence of different handoff parameters and procedures.

In section 2 a brief analysis of the capacity in CDMA systems for both up- and downlink is made. In section 3 we consider a general handoff model and obtain an analytical solution for the change in capacity due to soft-handoff. In section 4 we use the handoff procedure and parameters defined in IS95 , and obtain results through simulation. A brief conclusion is presented in section 5

\section{CAPACITY IN CDMA SYSTEMS}

The capacity of CDMA systems has to be investigated separately for the up- and downlink, due to their different characteristics, which may be summarized as follows:

- In the downlink all users are synchronized, which allows the use of orthogonal codes. It is true that due to multipath propagation, the orthogonality cannot be guaranteed at reception, yet nevertheless some performance gain can be expected. In the uplink, a chip synchronization of every user would be very difficult to implement, since each user has a different varying propagation delay, and random codes are therefore used.

- Power control is a more important issue in the uplink, since the signals from different users withstand different 
path loss and fading. This is not the case in the downlink, where there is a single source and only a power allocation procedure is generally implemented.

- In the IS-95 standards coherent demodulation is employed in the downlink only, relying on the transmission of a pilot signal by the base station. In the uplink a noncoherent modulation technique is employed. This means that a higher signal-to-interference ratio is required in the uplink than in the downlink for the same desired bit error rate.

\subsection{Uplink Capacity}

An analytical estimation of the uplink Erlang capacity of a CDMA system was presented in [7] considering inter-cell interference and imperfect power control. The capacity is defined as the traffic level for which a certain outage probability requirement is satisfied. It is assumed that an outage occurs when the interference power spectral density at the receiver is greater than a certain threshold $I_{0}$. The condition that outage does not occur for a data rate $R$ and $k$ users in a single cell is given by:

$$
\sum_{i=0}^{k} v_{i} E_{b_{i}} R+N_{0} W \leq I_{0} W
$$

where $E_{b_{i}}$ is the bit energy of the $i$-th user, $v_{i}$ ia a Bernouilli random variable with $\operatorname{Pr}(n=1)=\rho$ representing the voice activity of the $i$-th user, $N_{0}$ is the white noise power spectral density and $W$ is the transmission bandwidth.

Defining $e=E_{b} / I_{0}$, equation (1) can be rewritten as

$$
Z=\sum_{i=1}^{k} v_{i} \varepsilon_{i} \leq \frac{W}{R}(1-\eta)
$$

where $\eta=N_{0} / I_{0}$. A typical value for this parameter, also used in this paper is $\eta=0.1$ [7]

The received signal-to-interference ratio $\varepsilon_{i}$ for user $i$ is modeled by a log-normal random variable with mean $m_{x}=7$ $\mathrm{dB}$ and standard deviation $s_{x}=2.5 \mathrm{~dB}$.

Since $Z$ is the sum of $k$ independent random variables with same distribution, an approximation by the central limit theorem can be made as long as $k$ is large. In that case, $Z$ can be assumed to have a normal distribution and the outage probability depends only on its mean and variance. Defining $Z^{\prime}=Z e^{-\beta m}$, where $\beta=0.1 \ln 10$, the outage probability for a one cell system is given by:

$$
P_{\text {out }} \approx Q\left[\frac{A-E\left(Z^{\prime}\right)}{\sqrt{V A R\left(Z^{\prime}\right)}}\right]
$$

where $Q($.$) is the modified complementary error function,$

$$
A=\frac{\left(\frac{W}{R}\right)(1-\eta)}{e^{\beta m_{x}}}
$$

and for a traffic level $\lambda / \mu$ Erlang

$$
E\left(Z^{\prime}\right)=\frac{\lambda}{\mu} \rho e^{\left(\beta \sigma_{x}\right)^{2} / 2}
$$

$$
\operatorname{Var}\left(Z^{\prime}\right)=\frac{\lambda}{\mu} \rho e^{2\left(\beta \sigma_{*}\right)} .
$$

The interference from the neighbor cells is equivalent to increasing the interference by the factor $(1+f)$, where $f$ is the other-cell interference factor, typically 0.55 for a path loss exponent $n=4$ and a shadowing standard deviation $\sigma=$ $8 \mathrm{~dB}$ [7]. It can be included in this analysis if we substitute the equivalent traffic $(\lambda / \mu)^{\prime}=(\lambda / \mu)(1+f)$ in equations (5).

The use of soft-handoff has little influence on the uplink capacity, since the mobile station will transmit the same signal no matter how many base stations it is in contact with. It is argued in [1] that the uplink capacity will be increased by the use of soft-handoff, since the mobile station will always be controlled by the best server, and hence transmit with less power. This, however can be the case also with hard handoff, and is not necessarily true with soft-handoff, but depends on the handoff algorithm. Anyway, this would affect the interference factor $f$, and the value of 0.55 has been calculated with the assumption of control by the best server.

\subsection{Donwlink Capacity}

The estimation of the downlink capacity is not as straightforward as for the uplink. Here we follow the development in [1].

Suppose that a fraction $1-\beta$ of the total power transmitted by any base station is allocated to paging and broadcast channels, then each of the $k_{u}$ users in this cell receive a fraction $\beta \phi_{i}$ of the transmitted power, under the condition

$$
\sum_{i=1}^{k_{4}} \phi_{i} \leq 1
$$

We consider that the interference from the users in the same cell is reduced by an orthogonalization factor $h$. In case perfectly orthogonal codes are used $h=0$, but, as already mentioned, due to multipath propagation, full orthogonality cannot be maintained at the receiver, so that a value $h \leq 1$ should be employed (A value $h=1$ would be employed for random codes). Now let the total power from base station $j$ received by user $i$ be $S_{R_{j i}}$ and suppose that user $i$, controlled by base station 1 receives interference from $J$ base stations (the background noise can be ignored, since power saving is not as critical as in the uplink and hence the transmitted power is usually much higher than the noise power). Then, the bit energy to interference power spectral density ratio for the $i$-th user can be written as

$$
\left(\frac{E_{b}}{I_{0}}\right)_{i}=\frac{\beta \phi_{i} S_{R_{1 i}} / R}{\left(h+\sum_{j=2}^{J} S_{j i}\right) / W}
$$

The system is deemed to be in outage if there is no solution to equations (6) and (7) such that the required $E_{b} / I_{0}$ can be sustained for all users. Assume that the same ratio of bit energy to interference density $\Gamma_{D L} \mathrm{~L}$ is required by every user. Remembering that the users are active intermittently and inverting equation (7) we reach the following expression for the outage probability 


$$
\begin{aligned}
P_{\text {out }} & =\operatorname{Pr}\left(\sum_{i=1}^{K_{u}} \phi_{i}>1\right) \\
& =\operatorname{Pr}\left[\sum_{i=1}^{K_{u}} v_{i}\left(h+\sum_{j=2}^{J} \frac{S_{R_{j i}}}{S_{R_{2 i}}}\right)>\frac{\beta W / R}{\Gamma_{D L}}\right]
\end{aligned}
$$

In [1] the Chernoff bound on the outage probability is calculated, but an estimation based on the central limit theorem is also feasible here. Equation (8) can be rewritten as

$$
P_{\text {out }}=\operatorname{Pr}\left[\sum_{i=1}^{K_{u}} v_{i}\left(h+y_{i}\right)>\beta K_{0}\right]
$$

where

$$
\begin{gathered}
y_{i}=\sum_{j=2}^{J} \frac{S_{j i}}{S 1 i} \\
K_{0}=\frac{W / R}{\Gamma_{D L}}
\end{gathered}
$$

Now let $z_{i}=v_{i}\left(h+y_{i}\right)$, them the outage probability can be expressed as

$$
\begin{gathered}
P_{\text {out }}=\operatorname{Pr}\left[\sum_{i=1}^{K_{u}} z_{i}>\beta K_{0}\right] \Longrightarrow \\
\left.P_{\text {out }}=\sum_{k=0}^{\infty} \operatorname{Pr}\left[k_{u}=k\right] \operatorname{Pr}\left[\sum_{i=1}^{K_{u}} z_{i}>\beta K_{0}\right] K_{u}=k\right]
\end{gathered}
$$

Soft-handoff has a great influence in the downlink capacity, since the same signal will be transmitted by several base stations simultaneously, increasing the interference. This may be alternatively viewed as an increase in the effective traffic level in each cell. Let us define the parameter $g$ as the average number of base stations that are simultaneously in communication with any single user at any given time. If the actual traffic level is $\lambda / \mu$ per cell, we may say that the effective traffic level will be $(\lambda / \mu) g$ per cell on the downlink

We can thus assume that the number of signals transmitted in a cell is a Poisson random variable with parameter $g(\lambda / \mu)$. For high traffic levels the probability that few signals are transmitted is low and, by applying the central limit theorem, we can obtain the following approximation for the outage probability

$$
P_{\text {out }} \approx \sum_{k=0}^{\infty} \frac{(g \lambda / \mu)^{k} e^{-g \lambda / \mu}}{k !} Q\left(\frac{\beta K_{0}-k \bar{z}}{\sqrt{k} \sigma_{z}}\right)
$$

where

$$
\bar{z}=E\left[x_{i}\right]=\rho\left(h+E\left[y_{i}\right]\right)
$$

$\sigma_{z}^{2}=\operatorname{Var}\left[z_{i}\right]=\rho\left(h^{2}+2 h E\left[y_{i}\right]+E\left[y_{i}^{2}\right]\right)-\rho^{2}\left(h+E\left[y_{i}\right]^{2}\right)$
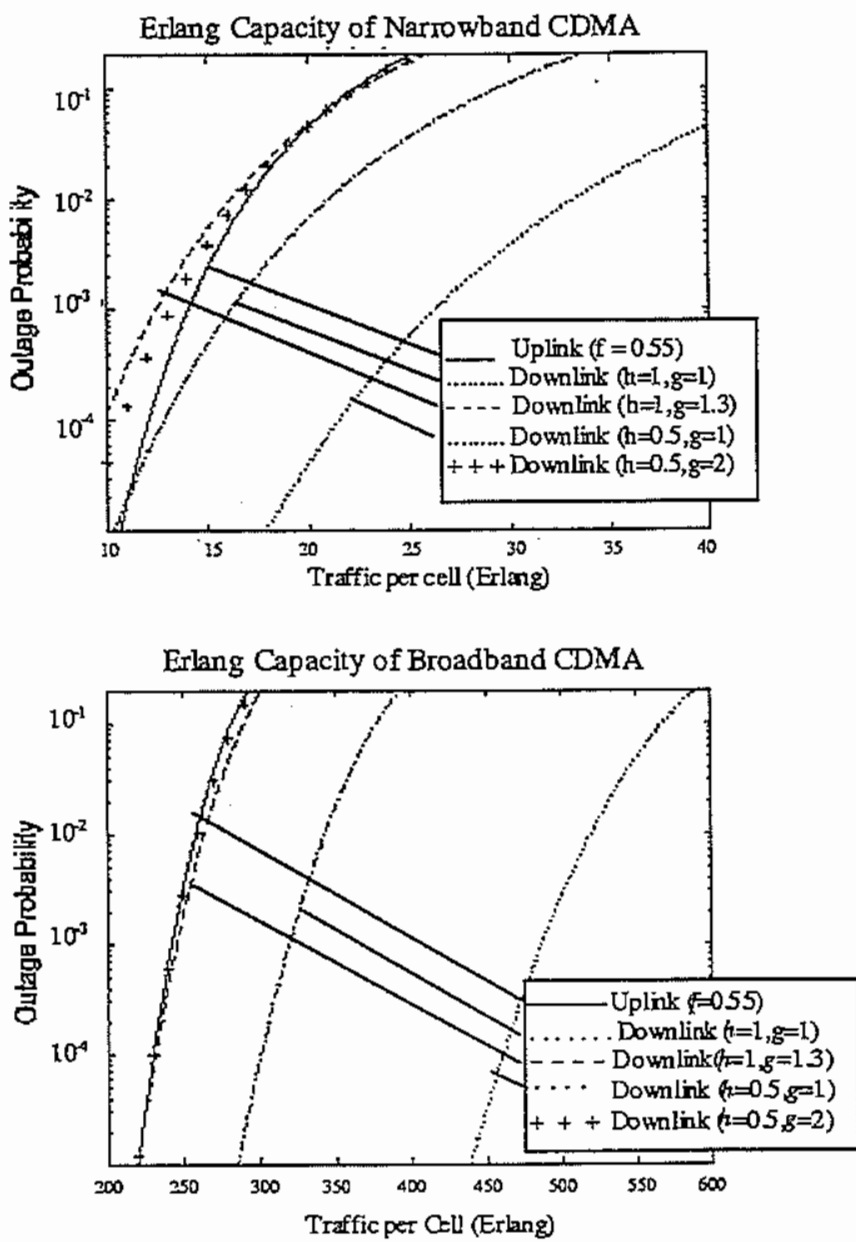

Figure 1: CDMA system capacity.

An analytical solution for $E\left[y_{i}\right]$ and $E\left[y_{i}^{2}\right]$ is very difficult to obtain, but simulation results for a path-loss exponent $n=4$ and a shadowing standard deviation $\sigma=8 \mathrm{~dB}$ indicate values of $E\left[y_{i}\right]=0.5125$ and $E\left[y_{i}^{2}\right]=1.025$. With these values we can numerically calculate the outage probability according to equations (12) and (13). Some results are shown in Fig. 1.

Fig. 1 shows curves of outage probability versus traffic per cell for the up- and downlink It can be seen that the capacity in the downlink is much higher than in the uplink, specially if soft-handoff is not taken into account. It can be said that the uplink is the limiting factor in the system capacity, as usually stated [7]. It is however clear from the above analysis, that depending on the value of $\mathrm{g}$, the downlink may limit the system capacity. If we consider non-orthogonal codes in the downlink a factor $g$ up to 1.3 can be accepted without capacity losses. With orthogonal codes the downlink capacity is much higher, even if full orthogonality is not maintained at the reception. In Fig.1 for example, for a orthogonality gain $h=0.5$ the allowable value of $g$ was increased to 2 . This however has to be viewed carefully, since the gain obtained with orthogonal codes is difficult to quantify and is heavily dependent from the channel model. It can be also noticed that the results are similar both for the narrow and for the 


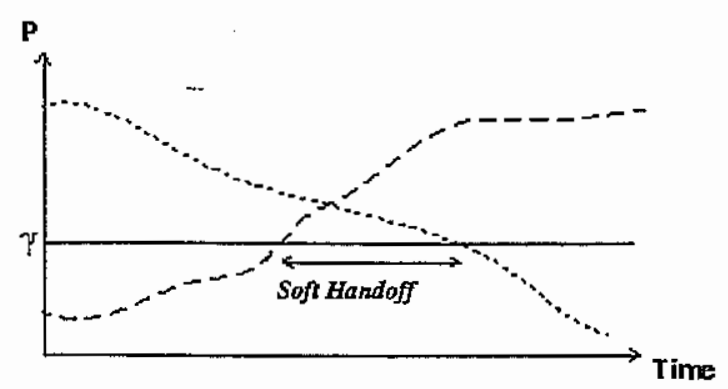

Figure 2: General handoff algorithm.

wide band cases.

It is desirable that many users be in contact with more than one base station, providing receiver diversity and seamless handoff as often as possible. On the other hand the average number of base stations in contact with a single mobile station cannot be too high, or capacity losses can be expected. Based on the above results, we assume that values of $\mathrm{g}$ between 1.3 and 1.4 should be aimed at. In the following sections we address the problem of determining the value of $g$ and how this factor is affected by different handoff schemes and parameters.

\section{GENERAL HAND-OFF MODEL}

In this section a general handoff method will be investigated analytically. Let us furst consider a two cell system. We assume that a mobile will be in soft-handoff with both cells if the reception level of both pilots is above a certain threshold. If we assume that ail pilots are transmitted with the same power, we may compare only the fading between the base stations and the subscriber. Letting $\alpha_{i}$ be the value of the fade between base station $i$ and the mobile station, we may then consider that a mobile will be in soft-handoff if

$$
\alpha_{1}, \alpha_{2}>\gamma
$$

where $\gamma$ is the threshold value. This is illustrated in Fig 2

We consider the propagation model used in [1], where the fading between a subscriber in a position with coordinates $(x, y)$ and base station $i$, in $\mathrm{dB}$, is given by

$$
\alpha_{i}(x, y)=-10 m \log \left[r_{i}(x, y)\right]+\alpha \xi+b \xi_{i}
$$

where $r_{i}(x, y)$ is the distance between $(x, y)$ and the base station $i, m$ is the path loss exponent, $\xi$ and $\xi_{i}$ are independent Gaussian random variables with variance $\sigma^{2}$ and zero mean, that represent the shadowing effect; $\xi$ is the same for every cell, and $\xi_{i}$ is a shadowing component relative to the $i$-th cell; $a$ and $b$ are parameters such that $a^{2}+b^{2}=1, a \leq 1$.

If we consider that the mobile location coordinates $(x, y)$ are random variables with joint probability density function $f_{x y}(X, Y)$ in a certain region $S_{0}$, the probability $P_{S H}$ that a subscriber is in soft-handoff is given by

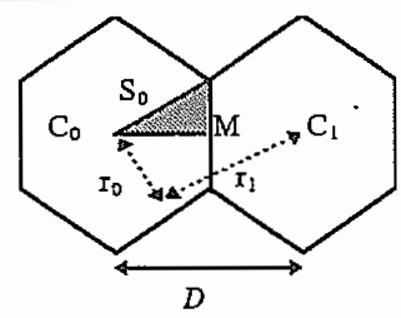

Figure 3: Two cells system

$$
P_{S H}=\iint_{S_{0}} f_{x, y}(X, Y) \cdot \operatorname{Pr}\left[\alpha_{0}, \alpha_{1}\right] d X d Y
$$

where

$\operatorname{Pr}\left[\alpha_{0}, \alpha_{1}\right]=\operatorname{Pr}\left[\alpha_{0}(x, y)>\gamma, \alpha_{1}(x, y)>\gamma \mid(x, y)=(X, Y)\right]$

Due to the symmetry of the problem, we may consider only the mobiles located in $\mathrm{S} 0 S_{0}$ in Fig. 1. We also assume a unitary distance from the center of a cell to the border between cells, so that the mean signal fading will be $0 d B$ in relation to both cells for a user located in $M$

In order to enable an analytical solution, we make $a=0$ and $b=1$, so that the shadowing fades for different cells are independent. Considering that the subscribers are uniformly distributed within $\mathrm{S} 0$, and taking $\mathrm{M}$ as the origin, we have

$$
P_{S H}=\int_{-1}^{0} \int_{0}^{(x+1 / \sqrt{3}} 2 \sqrt{3} Q\left(p_{0}(x, y)\right) Q\left(p_{1}(x, y)\right) d x d y
$$

where

$$
\begin{aligned}
& p_{0}(x, y)=\frac{\gamma+10 m \log r_{0}(x, y)}{\sigma} \\
& p_{1}(x, y)=\frac{\gamma+10 m \log r_{1}(x, y)}{\sigma}
\end{aligned}
$$

This analysis can be easily extended to a system with three or more cells. In this case the probability that a mobile is in soft-handoff with all $\mathrm{N}$ cells is given by

$$
P_{S H_{N}}=\int_{-1}^{0} \int_{0}^{(x+1) / \sqrt{3}} 2 \sqrt{3} \prod_{k=0}^{N-1} Q\left(p_{k}(x, y)\right) d x d y
$$

where

$$
p_{k}(x, y)=\frac{\gamma+10 m \log r_{k}(x, y)}{\sigma}
$$

The traffic increase $g$ due to soft handoff is calculated as the average number of base stations in soft handoff. We assume that the probability that the user is in communication with more than three base stations at the same time is very small, 


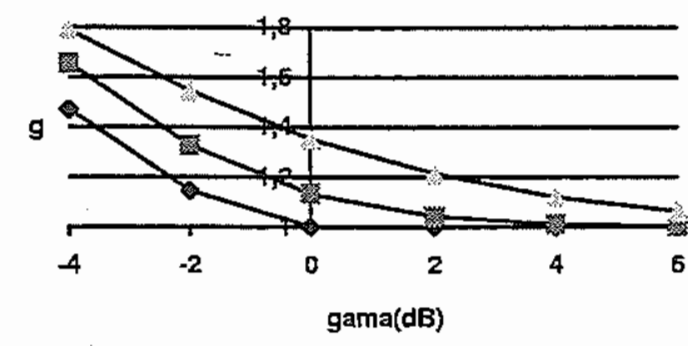

$-\operatorname{sigma}=0 \mathrm{~dB} \rightarrow-\operatorname{sigma}=4 \mathrm{~dB}-\operatorname{sigmn}=8 \mathrm{~dB}$

Figure 4: Values of $\mathrm{g}$ for differentes parameters

and calculate the value of $g$ for a three cell system, based on equations (17) and (18), as follows.

$$
\begin{aligned}
g= & 3 x \operatorname{Pr}[\mathrm{SH} \text { among all } 3 \text { cells }]+\operatorname{Pr}[\mathrm{No} \mathrm{SH}] \\
& +3 x \operatorname{Pr}[\mathrm{SH} \text { between any } 2 \text { cells }] \\
= & 3 x P_{\mathrm{SH}_{3}}+1-\left(P_{\mathrm{SH}_{3}}+3\left(P_{\mathrm{SH}_{2}}-P_{\mathrm{SH}_{3}}\right)\right. \\
& +2 x 3\left(P_{\mathrm{SH}_{2}}-P_{\mathrm{SH}_{3}}\right) \\
= & 3 P_{\mathrm{SH}_{2}}-P_{\mathrm{SH}_{3}}+1
\end{aligned}
$$

where $P_{\mathrm{SH}_{3}}$ and $P_{\mathrm{SH} 2}$ are respectively the probability of soft-handoff among 3 and 2 ceils. The results are shown on Figure [?], assuming $m=4$. We can see that the choice of the handoff threshold $\gamma$ is highly dependent on the shadowing variance.

\section{HANDOFF ACCORDING TO THE IS 95 STANDARD}

In this section we evaluate the reduction in the traffic capacity due to soft handoff in IS-95 CDMA. According to the IS95 standard, different base stations transmit a different pilot signals, which are in fact based upon the same pseudo-noise sequence, but with different delays. These pilot signals are constantly monitored by each mobile station, and the handoff is based on measures of the downlink signal-to-interference ratio. The algorithm defined in the IS-95 relies on a set maintenance procedure. All the pilots in the system are partitioned in the following sets:

- Active Set : contains the pilots of every base station in simuitaneous communication with the subscriber.

- Candidate Set: contains all pilots whose signals are strong enough for a good communication, and are therefore candidate to become active.

- Neighbor Set: contains the pilots of every base station that is adjacent to the main station, but whose signal is not strong enough for communication.

- Remaining Set : contains the pilots of every other base station in the system.

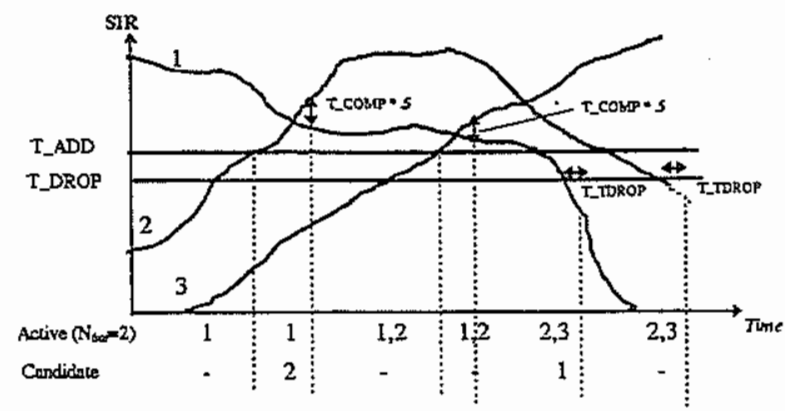

Figure 5: Handoff algorithm in IS 95.

The above sets are constantly updated depending on the following parameters

- T_ADD: is the threshold level that a pilot signal must exceed, for it to be moved into the Candidate Set.

- T_DROP, T_TDROP: If the level of a pilot belonging either to the Active or Candidate Set drops below T_DROP for at least T_TDROP seconds, it is excluded from its present set.

- T_COMP : If the level of a pilot belonging to the candidate set exceeds the level of any pilot belonging to the Active Set by at least T_COMP * 0,5 dB, it will be included in the active set.

- $N_{6 m}$ : is the maximum number of components in the active set.

At the beginning of any call, the Active Set is initialized with the pilot having the greatest received power. The Candidate Set is initially empty, the Neighbor Set contains the pilots of every geographically neighbor cell, and the Remaining Set, of every remaining cell in the system. An example of a IS-95 handoff procedure is shown in Figure 5.

The pilot level is measured by the signal-to-interference $S_{P} / I$, where $S_{P}$ is the pilot signal received power and $I$ is the interfering power. Considering an $N$-cell system, all transmitting with the same power on the downlink, and assuming that a fraction $\beta$ of each base station's power is dedicated to the pilot signal, we have that the received level of the 0th cell pilot is given by

$$
\frac{S_{p}}{I}=\frac{\beta \alpha_{0}}{(1-\beta) \alpha_{0}+\sum_{i=1}^{N-1} \alpha_{i}}
$$

where $\alpha_{i}$ is the fading between the subscriber and the $i$-th cell.

In order to obtain the value $g$ we must obtain the average number of elements in the Active Set Due to complexity of the algorithm, this calculation has been done through computer simulation. We have considered tre same paropagation model presented in the previous section, but this time we have considered $a=b=1 / \sqrt{2}$. We have assumed that each mobile moves with a constant speed, which is a uniform random variable between $m 0$ and $100 \mathrm{~km} / \mathrm{h}$, and in a fixed direction, 

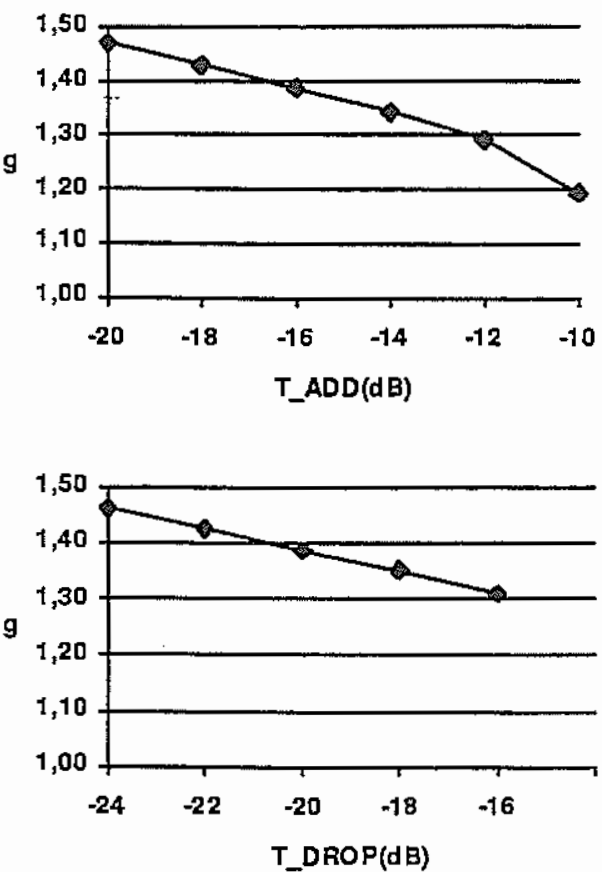

Figure 6: Value of $g$ in relation to T_ADD and T_DROP.

which is also a uniform random variable, between 0 and $2 \pi$. The duration of each call is an exponential random variable with mean : $\min$. Besides the model so far presented, we have also considered that the fading has a spatial correlation. This is simulated following the Gudmundson model [6], by which the fades from two points distanced $d$ from each other, in relation to the same base station, have a correlation given by:

$$
E\left[\alpha\left(p_{0}\right) \alpha\left(p_{1}\right)\right]=\sigma^{2} \exp \left(-d / d_{0}\right)
$$

where $d_{0}$ is a constant that determines the rate of decay with the distance.

In this simulation we have considered an urban environment, with $d_{0}=150 \mathrm{~m}, m=4, \sigma=8 \mathrm{~dB}$ and a distance of $4 \mathrm{~km}$ between adjacent base stations. Typical system parameters, based on field tests and simulations [4] are: T_ADD = $-16 \mathrm{~dB}, \mathrm{~T} \_\mathrm{DROP}=-20 \mathrm{~dB}, \mathrm{~T} \_\mathrm{COMP}=2,5 \mathrm{~dB}$ and $\mathrm{T}_{-} \mathrm{DROP}$ $=5 \mathrm{~s}$. These values were taken as reference in the simulations, and in each of the graphs shown below results for variation over one single parameter are shown.For variations over T_ADD, T_DROP is kept as T_ADD - $4 \mathrm{~dB}$. The results are shown in Figures 6,7 and 8

In Figure 6 we observe that the value of $g$ increases with the decrease in both T_ADD and T_DROP. This can be expected, since in this case the conditions for a pilot to be candidate for soft handoff are loosened. We must remember that these parameters represent the minimum level required for a good quality of transmission, and therefore there is not much margin for a reduction in these parameters below the typical values.

It can be noticed in Figure 7 that the value of $g$ increases with a decrease in T_COMP, since in this way the candidate pilots are more easily moved into the active set. We can also see that the lower the value of T_TDROP is, the smaller is


Figure 7: Value of $g$ in relation to $T_{-} T D R O P$ and $T_{-}$COMP.

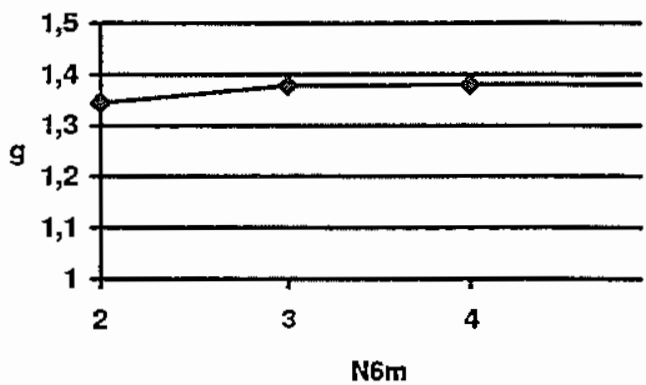

Figure 8: Value of $g$ in relation to $N_{6 m}$

the value of $g$ obtained, but the system is not very sensitive to variations in this parameter.

In Fig. 8 we observe that very little or no change occurs when we vary the parameter $N_{6 m}$ above 3 , and this is due to the fact that it is very unlikely that the mobile will be in softhandoff with more than 3 base stations. It can also be seen that for typical values the increase in the downlink effective traffic will be around $40 \%$.

\section{CONCLUSIONS}

We have seen in the results presented in this paper that the employment of soft-handoff may cause a substantial decrease in the downlink capacity of CDMA systems, and may even dominate the overall system capacity, since even though the capacity of the uplink is about $30 \%$ greater than that of the downlink, this extra capacity can be lost by an increase in the effective downlink traffic. Nevertheless, in order to have a more accurate picture of the problem, we must consider that the proportion of users in soft handoff will in practice vary in 
time, and the decrease in the downlink capacity may eventually be even higher than the one shown in this paper, which took only average values into account. It is therefore necessary to carefully choose the appropriate handoff parameters, so that the system capacity is not substantially decreased, while maintaining the performance improvements that can be obtained with soft-handoff. An estimation of the downlink capacity decrease for different handoff algorithms and parameters was carried out in this paper, and the results presented here may hopefully shed some light on this issue.

\section{REFERENCES}

[1] Viterbi, A.J., "CDMA - Principles of Spread Spectrum Communication", Addison-Wesley, 1995.

[2] TLA/EIA IS-95 Interim Standard, "Mobile Station - Base Station Compatibility Standard for Dual-Mode Wideband Spread Spectrum Cellular System", Telecommunication Industry Association, 1993.

[3] A.J. Viterbi, A.M. Viterbi, K.S. Gilhousen and E. Zehavi. "Soft Handoff Extends CDMA Cell Coverage and Increases Reverse Link Capacity", IEEE Journal on Selected Areas in Communications, Vol. 12, No 8, pp 12811288.

[4] M. Chopra, $\mathbb{K}$ Rohani and J.D. Reed, "Analysis of CDMA Range Extension due to Soft Handoff", Proceedings of the VTC 95, pp 917-921.

[5] Soleimanipour and G.H. Freeman, "A Realistic Approach to the Capacity of Cellular CDMA Systems", Proceedings of the VTC 96, pp 1125-1129.

[6] M. Gudmundson, "Correlation model for shadow fading in mobile radio systems", Electronic Letters, Vol. 27, No. 23, pp 2145-2146, November 1991.

[7] A.J. Viterbi and A.M. Viterbi, "Erlang Capacity of a Power Controlled CDMA System", IEEE Joumal on Selected Areas of Communications, August 1993, pp 892899.

André Noll Barreto - graduated in 1994 from the Pontifcal Catholic University of Rio de Janeiro (PUC/Rio), Brazil, and obtained a M.Sc. degree in Electrical Engineering from the same university in 1966, with work on the analysis of traffic in CDMA systems. He is currently working towards a doctor's degree at the Dresden University of Technology, Germany, where he is conducting research on GSM-based cordless telephone systems and wireless ATM networks.

João Celio Barros Brandão - graduated in 1969 from the Federal University of Juiz de Fora, Brazil, and received the M.Sc. degree in Electrical Engineering from the Pontifical Catholic University of Rio de Janeiro in 1973. Since 1971 he has been working as teacher and researcher at CETUC - Center for Studies in Telecommunications of PUC/Rio - where he is presently Associate Professor and Vice-Director. In 1977 and 1978 he worked at COMSAT Laboratories, USA, in the area of satellite communications and in 1991 he stayed at Hughes - Space and Communications Group, USA. In CETUC he has been working as researcher and supervisor in several research projects, in cooperation with $\mathrm{CPQD}$, the Center of Research of Telebrás in the area of interference between communication systems, digital transmission simulation and digital mobile cellular systems. He is a member of CBR4 , a technical committee designed by the Telecommunications Ministry for activities related to Satellite Communications in the Intemational Telecommunications Union. He is a founder member of the Sociedade Brasileira de Telecomaunicaçōes where he has been the Editor of the SBT Newsletter. 